\title{
ITER ALPHA PARTICLE DIAGNOSTICS USING KNOCK-ON ION TAILS
}

by

R.K. FISHER, C.W. BARNES, A. GONDHALEKAR, P.B. PARKS, J.M. McCHESNEY, A.L. ROQUEMORE, and M.N. ROSENBLUTH

\section{OCT 311933 \\ OSTI}

SEPTEMBER 1995 


\section{DISCLAIMER}

This report was prepared as an account of work sponsored by an agency of the United States Government. Neither the United States Government nor any agency thereof, nor any of their employees, makes any warranty, express or implied, or assumes any legal liability or responsibility for the accuracy, completeness, or usefulness of any information, apparatus, product, or process disclosed, or represents that its use would not infringe privately owned rights. Reference herein to any specific commercial product, process, or service by trade name, trademark, manufacturer, or otherwise, does not necessarily constitute or imply its endorsement, recommendation, or favoring by the United States Government or any agency thereof. The views and opinions of authors expressed herein do not necessarily state or reflect those of the United States Government or any agency thereof. 


\section{DISCLAIMER}

Portions of this document may be illegible in electronic image products. Images are produced from the best available original document. 


\title{
ITER ALPHA PARTICLE DIAGNOSTICS USING KNOCK-ON ION TAILS
}

by

\author{
R.K. FISHER, C.W. BARNES,,$^{\dagger}$ A. GONDHALEKAR, \\ P.B. PARKS, J.M. MCCHESNEY, A.L. ROQUEMORE, \\ and M.N. ROSENBLUTH ${ }^{\natural}$
}

This is a preprint of a paper presented at the Diagnostics for ITER International Workshop, August 28-September 1, 1995, and to be printed in Diagnostics for ITER.

\author{
Work supported by \\ U.S. Department of Energy \\ Grant No. DE-FG03-92ER54150 \\ and Contract No. DE-AC03-94SF20282 \\ ${ }^{\dagger}$ Los Alamos National Laboratory \\ ¥JET Joint Undertaking \\ "Princeton Plasma Physics Laboratory \\ "Also at University of California, San Diego
}

GENERAL ATOMICS PROJECT 3937

SEPTEMBER 1995 


\title{
ITER ALPHA PARTICLE DIAGNOSTICS
}

\section{USING KNOCK-ON ION TAILS}

\author{
R.K. Fisher, ${ }^{1}$ C.W. Barnes, ${ }^{2}$ A. Gondhalekar, ${ }^{3}$ P.B. Parks, ${ }^{1}$ \\ J.M. McChesney, ${ }^{1}$ A.L. Roquemore, ${ }^{4}$ and M.N. Rosenbluth ${ }^{5}$ \\ ${ }^{1}$ General Atomics, San Diego, California, U.S.A. \\ ${ }^{2}$ Los Alamos National Laboratory, Los Alamos, New Mexico, U.S.A. \\ ${ }^{3} \mathrm{JET}$ Joint Undertaking, Abingdon, Oxfordshire, U.K. \\ ${ }^{4}$ Princeton Plasma Physics Laboratory, Princeton, New Jersey, U.S.A. \\ ${ }^{5}$ Also at University of California, San Diego, California, U.S.A.
}

\section{INTRODUCTION}

Alpha particles will play a critical role in the physics and successful operation of ITER. Achieving fusion ignition requires that the $\alpha$-particles created by deuteriumtritium (D-T) reactions deposit a large fraction of their energy in the reacting plasma before they are lost. Toroidal field ripple can localize any alpha particle losses and cause first wall damage. Obtaining information on the $\alpha$-particles in ITER is a high priority diagnostic goal.

We have proposed a new method of measuring the fast confined $\alpha$-particle distribution in a reacting plasma. ${ }^{1}$ The same elastic collisions that transfer the alpha energy to the D-T plasma ions and allow fusion ignition will also create a high energy tail on the deuterium and tritium ion energy distributions. ${ }^{2,3}$ Some of these energetic tail ions will undergo fusion reactions with the background plasma producing neutrons whose energy is increased significantly above $14 \mathrm{MeV}$ due to the kinetic energy of the reacting ions. Measurement of this high energy tail on the D-T neutron distribution as a function of plasma minor radius would provide information on the alpha density profile with a time response equal to the ion slowing-down time ( $\sim$ few seconds).

Although this technique may provide only limited information on the $\alpha$-particle energy distribution, experimental studies of fast ions on existing tokamaks have shown that the observed slowing-down is essentially classical. Hence the $\alpha$-energy distribution is expected to be classical except in situations where the $\alpha$-confinement is poor. The confinement of $\alpha$ 's can be affected by ripple losses and a number of instabilities. ${ }^{4}$ Toroidal field ripple can cause both prompt orbit losses and stochastic ripple diffusion losses. Magnetohydrodynamic (MHD) activity, including fishbone instabilities, toroidal Alfvén eigenmodes (TAE), and sawtooth oscillations, may also affect alpha confinement. The diagnostic proposed here, by monitoring the confined 
alpha population, can provide valuable information on the confinement of fast alphas in a reacting plasma. Measurement of the confined alpha density profile will be especially important in ITER where it is needed to calculate the alpha heating profile. Studies of alpha heating will be an important objective of the ITER experiment.

\section{SIZE OF THE KNOCK-ON TAILS}

The first step is to calculate the size of the energetic plasma ion tail populations due to knock-on collision with alphas. Our results are reported in Ref. 1. Results of similar calculations are reported in Ref. 5. Using the classical slowingdown alpha distribution under ITER Conceptual Design Activity (CDA) conditions $T_{\mathrm{e}}=T_{\mathrm{i}}=17 \mathrm{keV}, n_{\mathrm{e}}=2 n_{\mathrm{D}}=2 n_{\mathrm{T}}=8 \cdot 10^{13} \mathrm{~cm}^{-3}$, we find the ion tail spectra given in Fig. 1. This figure shows the fractional tritium ion density per $\mathrm{MeV}$ versus the ion energy. A very similar result is obtained for the deuterium ion tail due to knock-on collisions with alphas. ${ }^{1}$ Figure 1 shows two curves, the result with only Coulomb scattering included and the result with nuclear plus interference forces included. Nuclear forces are important for these small impact parameter knock-on collisions. The alpha knock-on tail breaks out of the Maxwellian ion tail at ion energies above approximately $200 \mathrm{keV}$. At this energy the fractional tritium ion density $n_{\mathrm{t}}^{-1} d n_{\mathrm{t}} / d E \sim 10^{-3}$, falling relatively slowly to $10^{-4}$ at $\sim 800 \mathrm{keV}$ and $10^{-5}$ at $\sim 2 \mathrm{MeV}$. Also shown in Fig. 1 is the classical slowing-down distribution of $1 \mathrm{MeV}$ tritons from D-D fusion reactions occurring in this D-T plasma. Fortunately, the alpha-induced tail is larger than the $D-D$ triton population by over a factor of five, so that measurements reflecting the ion tail can be used to provide information on the alphas.

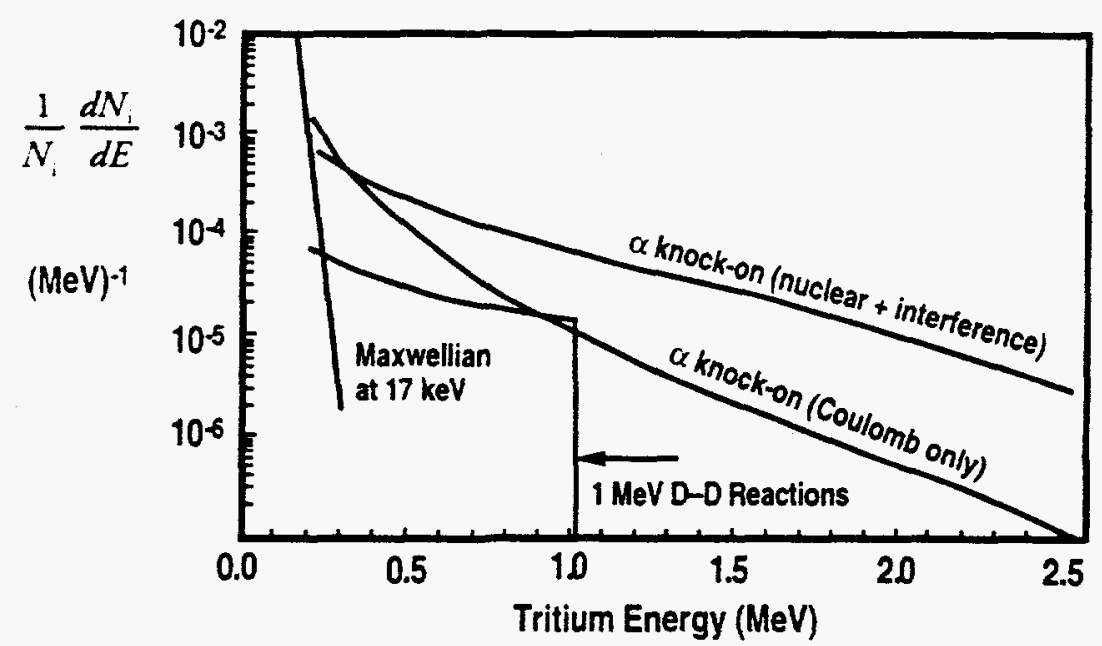

Figure 1. Calculated ion energy distributions due to alpha knock-on collisions under ITER conditions. $T_{e}=T_{\mathrm{i}}=17 \mathrm{keV}, n_{e}=2 \mathrm{n}_{\mathrm{D}}=2 n_{\mathrm{T}}=8 \cdot 10^{13} \mathrm{~cm}^{-3}$. Figure shows the fractional tritium ion density in units of $(\mathrm{MeV})^{-1}$. The effects of the knock-on collisions with Coulomb forces only and with nuclear plus interference forces are shown. Also shown is the classical $1 \mathrm{MeV}$ triton distribution from $D-D$ reactions. 
Next we calculate the $\mathrm{D}-\mathrm{T}$ neutron energy spectrum resulting from these energetic knock-on tail ions undergoing fusion reactions with their much more populous thermal counterparts. Because of their higher mass, the tritium tail ions contribute significantly more to the energetic neutron tail than do the deuterium tail ions. ${ }^{1}$ The neutron energy resulting from a triton of energy $E_{\mathrm{T}}$ colliding with a thermal $\mathrm{D}$ ion $\left(E_{\mathrm{D}} \ll E_{\mathrm{T}}\right)$ is given by $E_{\mathrm{n}}=\left\langle E_{\mathrm{n}}\right\rangle+\Delta E_{\mathrm{n}} \cos \theta$ where $\theta$ is the CM-frame angle of emission of the neutron with respect to the incident triton direction and

$$
\begin{aligned}
& \left\langle E_{\mathrm{n}}\right\rangle=\frac{Q}{1+m_{\mathrm{n}} / m_{\alpha}}+a E_{\mathrm{T}} \\
& \Delta E_{\mathrm{n}}=b\left(Q E_{\mathrm{T}}+d E_{\mathrm{T}}^{2}\right)^{1 / 2}
\end{aligned}
$$

where

$$
\begin{aligned}
& a=\frac{1}{\left(1+m_{\mathrm{n}} / m_{\alpha}\right)\left(1+m_{\mathrm{T}} / m_{\mathrm{D}}\right)}+\frac{m_{\mathrm{n}} m_{\mathrm{T}}}{\left(m_{\mathrm{T}}+m_{\mathrm{D}}\right)^{2}}, \\
& b=2\left[\frac{m_{\mathrm{T}} m_{\mathrm{n}}}{\left(m_{\mathrm{D}}+m_{\mathrm{T}}\right)^{2}\left(1+m_{\mathrm{n}} / m_{\alpha}\right)}\right]^{1 / 2}, \\
& d=\left(1+m_{\mathrm{T}} / m_{\mathrm{D}}\right)^{-1} .
\end{aligned}
$$

This result comes from conservation of energy and momentum. The $Q$ for the $\mathrm{D}-\mathrm{T}$ reaction is $17.6 \mathrm{MeV}$ so that

$$
E_{\mathrm{n}}=14.1 \mathrm{MeV}+0.44 E_{\mathrm{T}}+0.62\left[E_{\mathrm{T}}\left(17.6 \mathrm{MeV}+0.4 E_{\mathrm{T}}\right)\right]^{1 / 2} \cos \theta
$$

Since a tritium ion can receive up to $3.4 \mathrm{MeV}$ in a single collision with a $3.5 \mathrm{MeV}$ alpha, Eq. (4) indicates neutrons with energies up to $20.6 \mathrm{MeV}$ will result from $\alpha$ particle collision-induced tail ions.

Figure 2 shows the D-T neutron energy spectra calculated using Eq. (4) and the tritium ion distribution of Fig. 1 reacting with the bulk deuterium ions. ${ }^{1}$ The ion distributions were assumed to be isotropic in $\theta$. The vertical axis is the fractional neutron distribution $N_{\mathrm{DT}}^{-1} d N_{\mathrm{DT}} / d E_{\mathrm{n}}$ in units of $(\mathrm{MeV})^{-1}$, while the horizontal axis is neutron energy. The neutron tail is smaller than the ion tail due to the drop in the $\mathrm{D}-\mathrm{T}$ fusion cross-section with tritium ion energy above $E_{\mathrm{T}} \sim 180 \mathrm{keV}$.

Other possible sources of high energy neutrons in a fusion plasma include thermal broadening of the $\mathrm{D}-\mathrm{T}$ neutron emission and reactions involving fast ions from neutral beam or ICRF heating. Alpha knock-on effects begin to dominate the thermal broadening of the D-T neutron spectrum above neutron energies of $\sim 15.5 \mathrm{MeV}$. Beam-beam reactions will not produce neutrons above $16.0 \mathrm{MeV}$ at beam energies of up to $140 \mathrm{keV}$ so will not be a problem on TFTR or JET. Beam-target neutrons will also not be a problem at these beam energies according to our calculations. If ITER uses neutral beams for heating or current drive at energies $\sim 0.5$ to $1 \mathrm{MeV}$, the knockon neutron technique would remain useful during the latter stages of the heating phase of ITER and during the ignited burn phase, after the beams have been turned off. ICRF heating can easily produce energetic tails on minority ion distributions but will not produce a significant energetic tail on the majority deuterium or tritium ion distributions unless the plasma density is low $\left(\sim\right.$ a few $\left.10^{13} \mathrm{~cm}^{-3}\right)$. The question of 


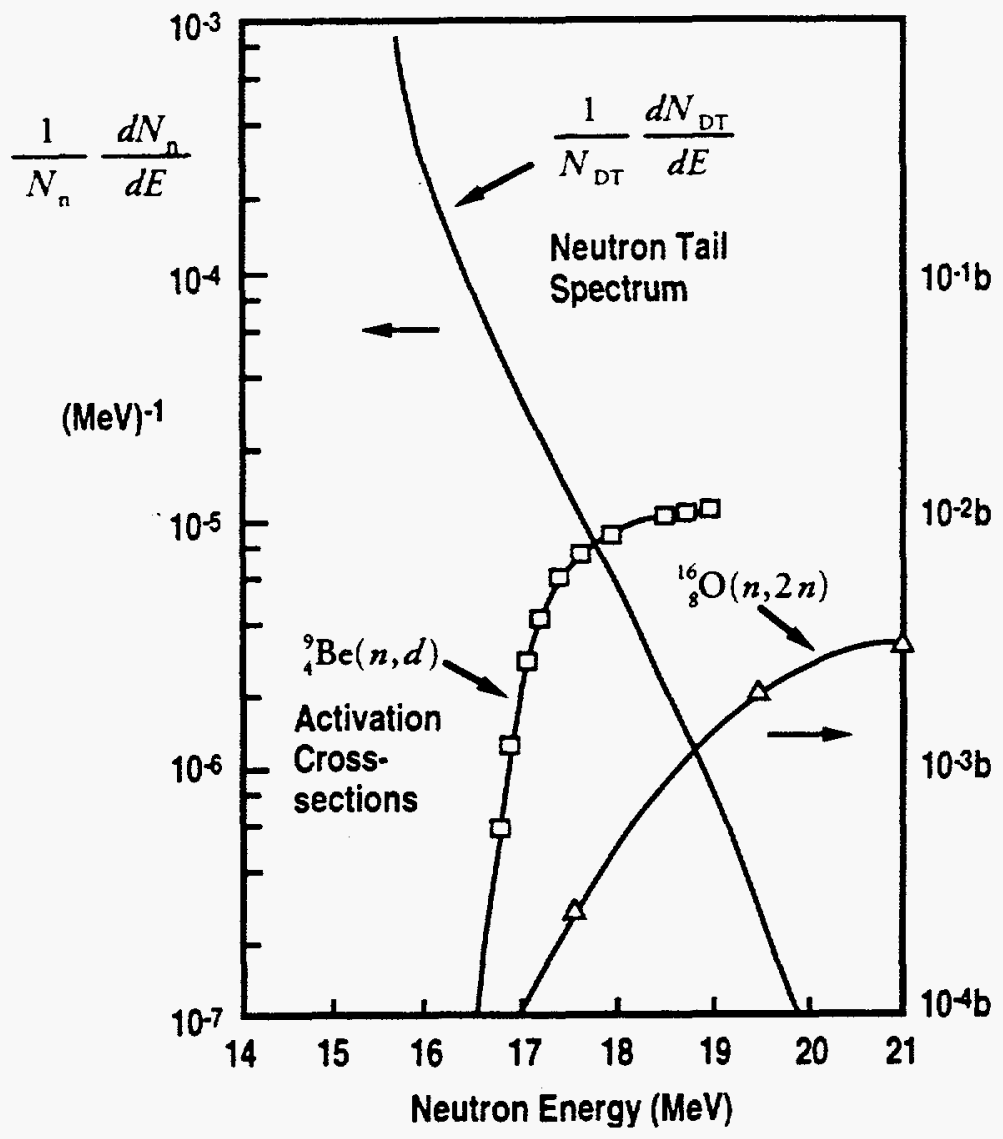

Figure 2. Calculated D-T neutron energy spectrum for the tritium ion distribution of Fig. 1 reacting with thermal deuterium ions. Also shown are the neutron activation cross-sections for the ${ }^{9} \mathrm{Be}(n, d)$ and ${ }^{16} \mathrm{O}(n, 2 n)$ reactions.

whether ICRF heating will enhance the tail created by the alpha knock-on collisions needs to be examined. We expect the alpha-knock-on effects to dominate the neutron tail production above $16 \mathrm{MeV}$ under most plasma conditions on TFTR, JET, and ITER.

\section{NEUTRON THRESHOLD ACTIVATION DETECTORS}

One of the most straightforward and attractive methods of measuring the $D-T$ neutron tail is activation detectors. This method utilizes nuclear reactions which require that the incident neutron energy be above a threshold energy to produce the reaction. A very attractive candidate reaction is:

$$
n+{ }_{8}^{16} \mathrm{O} \rightarrow{ }_{8}^{15} \mathrm{O}+2 n,
$$

with a threshold energy of $16.7 \mathrm{MeV}$. Figure 2 also shows the ${ }^{16} \mathrm{O}(n, 2 n)$ cross-section ${ }^{6}$ as a function of neutron energy. The ${ }^{15} \mathrm{O}$ end product subsequently decays to ${ }^{15} \mathrm{~N}$ via positron emission with a half-life of $2.1 \mathrm{~min}$. 
By exposing an activation target containing oxygen to the $\mathrm{D}-\mathrm{T}$ neutron flux from a reacting plasma, only those neutrons with energies above $16.7 \mathrm{MeV}$ can excite the ${ }^{16} \mathrm{O}(n, 2 n)$ reaction. By only exposing the activation target for a few seconds, we can monitor the alpha-induced tail with a time response limited only by the energetic deuterium and tritium ion slowing-down times ( $\gtrsim 1 \mathrm{sec}$ in these plasmas). The target is then transported to a radiation-quiet area where the ${ }^{15} \mathrm{O}$ decay gammas are measured. The positron emitted by ${ }^{15} \mathrm{O}$ will annihilate with an electron inside the activation target producing two coincident $511 \mathrm{keV}$ gamma-rays emitted in opposite directions. Hence the activation target would be placed inside a split well detector which would require both gamma-rays to be detected in coincidence. Measuring the $511 \mathrm{keV}$ coincident gamma-rays that were observed to decay in a $2.1 \mathrm{~min}$ half-life would ensure that we were monitoring the desired ${ }^{16} \mathrm{O}(n, 2 n)$ activations, and allow discrimination against other background sources of gamma-rays. Background gammas from ${ }^{16} \mathrm{O}(n, p)$ reactions excited by the much larger flux of $14 \mathrm{MeV}$ neutrons would be a problem if not for the $7.4 \mathrm{sec}$ half-life of the resulting ${ }^{16} \mathrm{~N}$. The ${ }^{16} \mathrm{O}(n, 2 n)$ reactions resulting from the neutron tail will dominate the decay gamma spectrum $4 \mathrm{~min}$ after the exposure to the tokamak neutron flux. It will also be necessary to load the transport capsule used to transfer the oxygen activation target in a helium or other non-air atmosphere. Otherwise the $14 \mathrm{MeV}$ neutrons will produce ${ }^{14} \mathrm{~N}(n, 2 n)$ reactions with the air trapped inside the capsule. The resulting $\beta^{+}$decays of ${ }^{13} \mathrm{~N}$, which has a 10 minute half-life, would dominate over the ${ }^{16} \mathrm{O}(n, 2 n)$ signal reactions from the knock-on neutron tail. Preliminary tests of this approach are underway on TFTR.

Using the calculated neutron spectrum and the activation cross-section in Fig. 2, we have calculated the expected signal levels for various tokamaks. The existing activation target transfer systems on TFTR and JET restrict the target volume to approximately $20 \mathrm{~cm}$. This size target exposed for $1 \mathrm{sec}$ during D-T operation of TFTR or JET at $4 \cdot 10^{18} \mathrm{n} / \mathrm{sec}$ would produce about 9,000 total ${ }^{16} \mathrm{O}(n, 2 n)$ activations. Hence the detected coincident gamma-ray signal rate would be approximately $50 / \sec E^{-t / 182 \sec }$ for a high-efficiency well detector. ITER at a neutron output of $4 \cdot 10^{20} \mathrm{n} / \mathrm{sec}$ would produce $2 \cdot 10^{4}$ total activations after a $1 \mathrm{sec}$ exposure yielding a detected gamma signal rate of $1.3 \cdot 10^{5} / \mathrm{sec} e^{-t / 182 \mathrm{sec}}$ for a $1000 \mathrm{~cm}^{3}$ target.

Using other target elements with different neutron energy thresholds could provide a cross-check on the results and also possibly provide some information on the $\alpha$ particle energy distribution. A classical slowing-down alpha distribution was assumed in the results of Figs. 1 and 2. For example, the reaction with beryllium

$$
{ }_{4}^{9} \mathrm{Be}+n \rightarrow{ }_{3}^{8} \mathrm{Li}+d,
$$

has a threshold energy of $16.3 \mathrm{MeV}$ and a cross-section ${ }^{6}$ about ten times larger than the ${ }^{16} \mathrm{O}(n, 2 n)$ cross-section as shown in Fig. 2. The resultant ${ }^{8} \mathrm{Li}$ decays via $\beta^{-}$ decay with a half-life of $0.84 \mathrm{sec}$. The short half-life means that the target transfer must be done as quickly as possible. The existing JET transfer system requires about $8 \mathrm{sec}$ but it may be possible to reduce this. ${ }^{7}$ A Cerenkov detector is attractive because it only produces a signal if the decaying $\beta^{-}$has a velocity greater than the velocity of light in the Cerenkov medium. Even high purity Be contains impurities such as boron, carbon, and oxygen which can be activated by the much larger flux of $14 \mathrm{MeV}$ neutrons and also result in $\beta^{-}$decays. Fortunately, we have calculated that the purest forms of Be should be useable if the Cerenkov detector is designed to detect only $\beta^{- \text {'s }}$ with energies above $10.4 \mathrm{MeV}$. The Cerenkov $\beta^{-}$energy threshold is controlled by the 
index of refraction in the chamber, which is determined by the gas pressure. Under ITER conditions, the calculated ${ }^{9} \mathrm{Be}(n, d)$ activation using Fig. 2 results in $5 \cdot 10^{5} / \mathrm{sec}$ decay $\beta^{-}$'s for a $20 \mathrm{~cm}^{3}$ Be target exposed for $1 \mathrm{sec}$. Since the half-life is only $0.8 \mathrm{sec}$ the activation rate quickly saturates at this level and then tracks the knock-on tail population with $\sim 1 \mathrm{sec}$ time resolution. Under JET and TFTR conditions, the corresponding signal size is $6 \cdot 10^{4} / \mathrm{sec}$. The signal drops to $\sim 70 / \mathrm{sec}$ after the $8 \mathrm{sec}$ transfer time required by the existing JET transfer system.

By installing a number of threshold activation detectors in a collimated neutron detector array, it may be possible to monitor the neutron tail size as a function of minor radius in the plasma. The neutron tail is linearly proportional to the local alpha density in the region viewed by the detector. Using the profiles of electron temperature and density measured by other diagnostics, we can then calculate the local alpha density producing the knock-on tail. Since the neutron emission profile is highly peaked, the neutron tail measured by each detector in the array will reflect the alpha density at the point of highest neutron emission in its view, i.e., the point where the detector sightline is tangent to the plasma flux surface.

\section{NEUTRON SPECTROSCOPY TECHNIQUES}

We have examined a number of other methods to observe the energetic $\mathrm{D}-\mathrm{T}$ neutron tail due to alpha knock-on collisions. A magnetic proton recoil (MPR) spectrometer similar to that discussed by J. Källne et al. ${ }^{8}$ has promise for measurement of the $\mathrm{D}-\mathrm{T}$ neutron tail. Ideally it would be designed to maximize the small neutron tail signal by increasing the detection efficiency at the expense of reducing the energy resolution. Hence it might differ somewhat from an MPR system optimized for ITER ion temperature and $\mathrm{D} / \mathrm{T}$ fuel density ratio measurements. This technique requires that the detector background due to neutrons and gamma rays from ITER be minimized using a combination of shielding and detectors with very low neutron and gamma ray sensitivity.

Another technique we are studying is neutron time-of-flight spectroscopy. Timeof-flight spectroscopy has been successfully used on JET to measure ion temperature. Figure 3 shows a high count rate time-of-fight spectrometer proposed for ITER by T. Elevant et al. ${ }^{9}$. The collimated incident DT neutron flux interacts with a thin $(0.3 \mathrm{~mm})$ polyethylene scattering foil oriented along the neutron path. Neutrons scattered at $45 \mathrm{deg}$ from probes in the foil are detected in the lower set of fast scintillator detectors $D_{2}$ which are approximately $5 \mathrm{~m}$ from the foil. The start signal is obtained by detecting the foil protons which are scattered upward at 45 deg into a linear array of fast scintillation detectors. Both the scattered neutrons and protons carry half the incident neutron energy, because of the 45 deg scattering angle. The projected overall neutron signal rate is $\dot{N}_{\mathrm{DT}} \sim 10^{6} / \mathrm{sec}$ at an incident neutron flux of $10^{9} / \mathrm{cm}^{2}$-sec. Using the results in Fig. 2, we can expect

$$
\frac{d N_{\text {tail }}}{d E} \sim 10^{-4} / \mathrm{MeV}\left(10^{6} / \mathrm{sec}\right) \sim 10^{2} / \mathrm{MeV} \text {-sec }
$$

counts in the neutron tail at $E_{n} \sim 16.5 \mathrm{MeV}$.

The expected time-of-flight spectrum is schematically shown in Fig. 4. The ion temperature broadened DT neutron peak appears above the flat random coincidence spectrum. On the high energy (short flight time) side of the spectrum, the knock-on tail neutrons appear above the flat random coincidence background when 


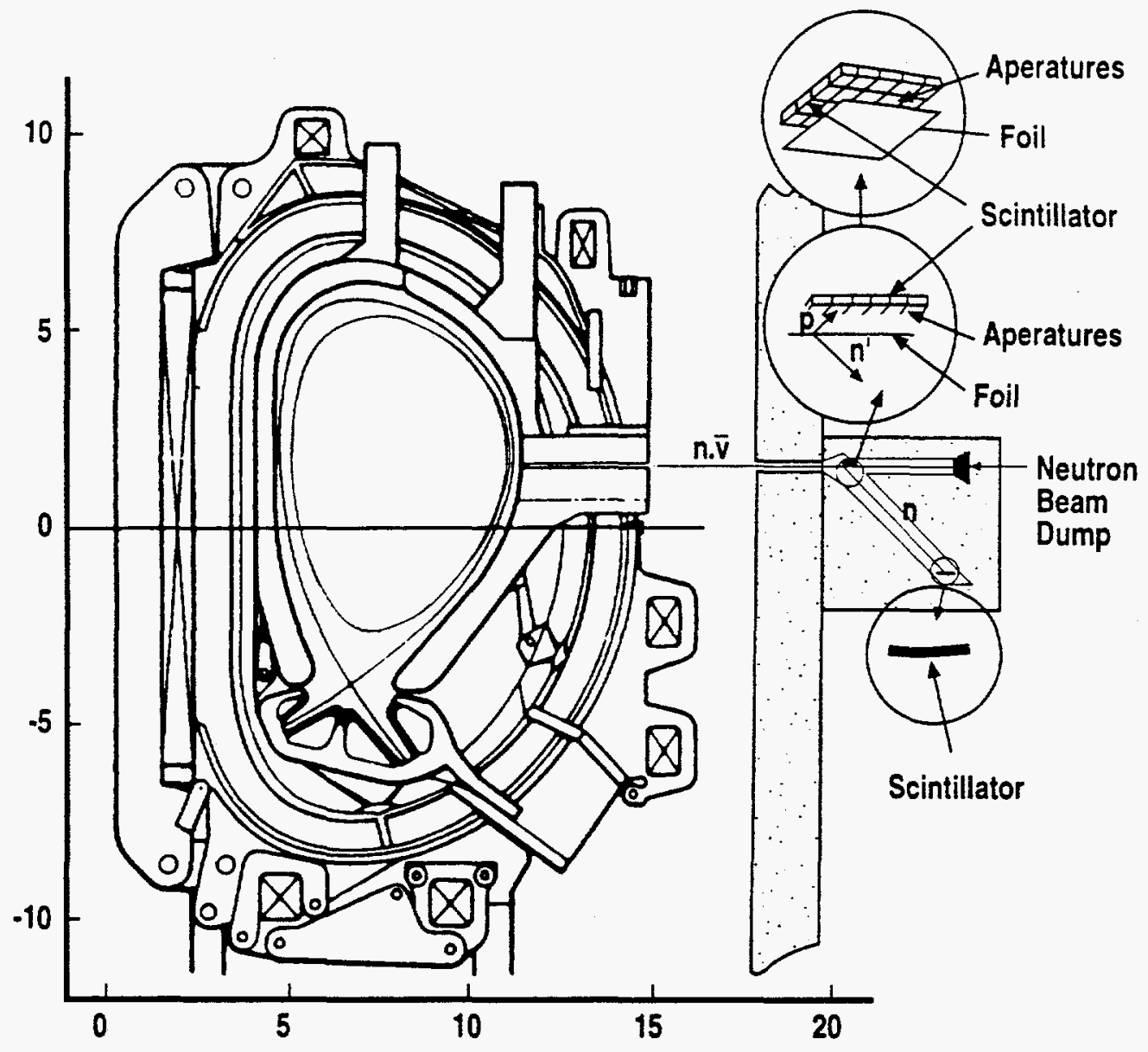

(m)

Figure 3. High count rate time-of-flight neutron spectrometer for ITER proposed by T. Elevant, Rev. Sci. Instrum. 66:881 (1995).

$$
N_{\text {tail }}=\dot{N}_{\text {tail }} \Delta t \gg\left(\dot{N}_{1} \dot{N}_{2} \tau \Delta t\right)^{1 / 2}
$$

where $\dot{N}_{\text {tail }}$ is the knock-on signal rate, $\Delta t$ is the data collection time, $\dot{N}_{1}$ and $\dot{N}_{2}$ are the random (background) counting rates in the start and stop detectors, and $\tau$ is the time window $\sim 1$ nsec. Hence it is very important to minimize the background counting rates, $\dot{N}_{1}$ and $\dot{N}_{2}$. The intrinsic backgrounds due to neutron scattering from the scattering foil will require integrating the data for $\sim 30 \mathrm{sec}$ to observe the knock-on tails.

The time-of-flight spectrometer described in Ref. 9 is designed for ion temperature measurements. It should be possible to sacrifice some energy resolution and reduce the required data collection time for knock-on tail measurements. For example, if the neutron detectors are moved closer to the scattering foil, both the signal rate $\dot{N}_{\text {tail }}$ and the intrinsic background rate $\dot{N}_{2}$ should increase. This should result in a reduction in the data collection time necessary for measuring the knock-on tail. Trade-offs are 


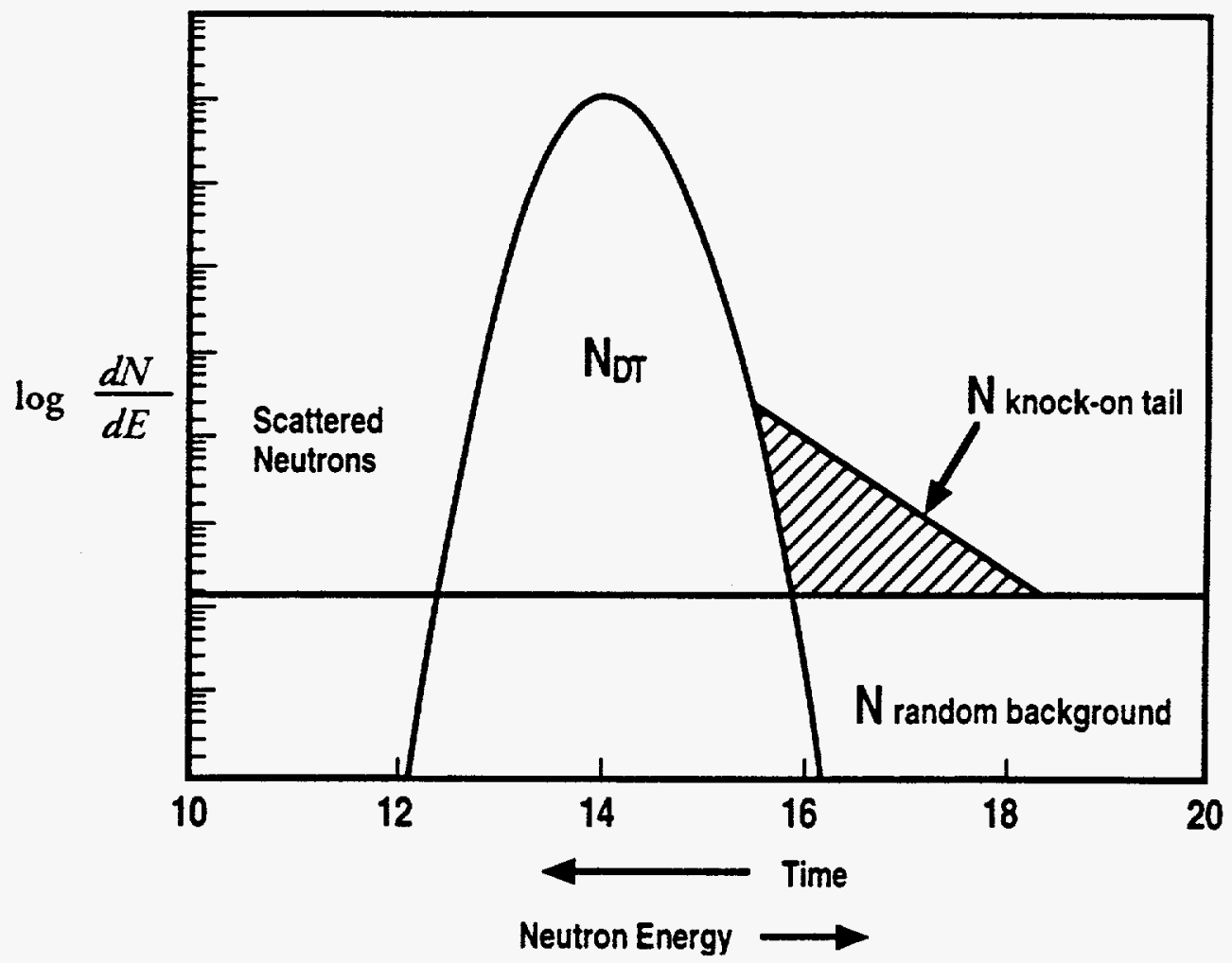

Figure 4. Expected time-of-flight neutron spectrum showing knock-on tail and "flat" background due to random coincidences.

also possible in the neutron scattering angle, proton detector distance and angular collimation, and foil thickness and composition.

Other neutron spectroscopy techniques under consideration as a result of this workshop include scintillating fiber neutron spectrometers ${ }^{10}$ and bubble chamber threshold detectors. ${ }^{11}$

\section{OBSERVING ION TAILS DIRECTLY}

In addition to measuring the $\mathrm{D}-\mathrm{T}$ neutron tail due to alpha knock-on collisions, it may be possible to directly measure the knock-on plasma ion tails. Experiments on JET have shown that energetic hydrogen neutrals are created when ICRF heating generates $\mathrm{H}$-ion tails and these $\mathrm{MeV}$ tail ions subsequently charge exchange with single electron impurity ions $\mathrm{Be}^{3+}$ and $\mathrm{C}^{5+}$ in the center of $\mathrm{JET} .{ }^{12}$ We have observed energetic $\mathrm{H}$ neutrals during similar experiments on TFTR, and have also observed tritium neutrals from $1 \mathrm{MeV}$ tritons produced in D-D reactions in TFTR. JET experiments have also resulted in observations of high energy tritium neutrals from $1 \mathrm{MeV}$ tritons interacting with single electron impurity ions during $\mathrm{D}-\mathrm{D}$ experiments. Since the predicted $\mathrm{D}$ and $\mathrm{T}$ energetic ion tails due to alpha knock-on collisions are somewhat larger than these $1 \mathrm{MeV}$ triton populations, we can expect to observe measurable high energy neutral signals due to alpha knock-on collisions during D-T 
operation of JET. This assumes we add neutron shielding to the JET neutral particle analyzer to keep the D-T neutron background near present $D-D$ levels. This should be possible since we have achieved a reduction of more than a factor of 100 in D-T neutron background by adding neutron shielding to a nearly identical Ioffe analyzer on TFTR.

Hence it may also be possible to observe the $\mathrm{D}$ - and T-ion tails due to alpha collisions as they also can pick up a single electron from impurity ions. Calculations show this technique should produce neutral signals on JET comparable to those produced for alpha observations while not requiring a helium diagnostic beam. Reionization of the newly created tritium neutrals as they exit the plasma will require correction of the measured spectra. For example, approximately $50 \%$ of the $1 \mathrm{MeV}$ tritons created at $r / a \sim 0.5$ will escape to the edge of ITER. Tests of the direct ion tail measurements using a high energy neutral particle analyzer are planned on JET in collaboration with JET scientists.

\section{CONCLUSIONS}

Measurement of knock-on tails should provide valuable information on alpha particles in ITER. Direct measurements of the energetic ion tails would provide information on both the alpha population and energy spectrum, and could be done using the same high energy neutral particle analyzers proposed for alpha charge exchange measurements using neutral beams or pellets.

Measurements of the energetic neutron tail on the DT neutron emission from ITER offers a very attractive approach to alpha particle diagnostics. It is nonperturbing to the plasma, relatively low cost, and requires no vacuum penetrations. A collimated array of neutron threshold activation detectors can be used to provide information on the alpha density profile. Time-of-flight and/or magnetic proton recoil neutron spectroscopy should provide information on the alpha energy spectrum as well as the alpha population in ITER.

It would be highly desirable to test these approaches during $\mathrm{D}-\mathrm{T}$ operation of TFTR or JET. Preliminary tests of the oxygen neutron threshold activation system are planned for TFTR, while tests of the direct ion tail measurements using charge exchange neutrals are proposed on JET.

\section{ACKNOWLEDGMENTS}

We would like to thank S.S. Medley, M. Petrov, and H.H. Duong for their efforts on the observations of $1 \mathrm{MeV}$ tritons and MeV hydrogen ICRF tail ions in TFTR.

This is a report of research and work sponsored by the U.S. Department of Energy under Grant No. DE-FG03-92ER54150 and Contract No. DE-AC03-76CH03073.

\section{REFERENCES}

1. R.K. Fisher et al., Fast alpha particle diagnostics using knock-on ion tails, Nucl. Fusion 34:1291 (1994).

2. P.B. Parks et al., private communication (General Atomics, 1991). 
3. D. Ryutov, Energetic ion propulsion formed in close collision with fusion alpha-particles, Physica Scripta 45:153 (1992); P. Helander et al., Formation of hot ion population in fusion plasmas by close collisions with fast particles, Plasma Phys. and Contr. Fusion 35:363 (1993). J. Källne et al., Alpha particle information from neutron observations, Fresion Technol. 25:341 (1994).

4. W. W. Heidbrink and G.J. Sadler, The behavior of fast ions in tokamak experiments, Nucl. Fusion 34:535 (1994).

5. G. Gorini et al., Alpha particle kinetic effects in the neutral emission of burning DT plasmas, Rev. Sci. Instrum. 66:936 (1995).

6. Victoria McLane et al., Neutron Cross Sections, Academic Press, San Diego (1988), Vol. 2, BNL-325.

7. O.N. Jarvis, private communication (JET, 1994).

8. J. Källne, Neutron spectroscopy for TFTR, talk I14 of this meeting; J. Källne et al., On the possibility of neutron spectrometry for determination of fuel in densities in DT plasmas, Rev. Sci. Instrum. 62:2871 (1991); see also Ref. 5.

9. T. Elevant, A neutron spectrometer for ITER, Rev. Sci. Instrum. 66:881 (1995); also Ion temperature profile measurements in ITER by means of neutron spectroscopy, this meeting.

10. T. . Elevant, Scintillating fiber neutron spectrometer, this meeting.

11. S.V. Trusillo, Neutron image system for neutron diagnostic of ITER plasma, this meeting.

12. A. Gondhalekar et al., Measurements of MeV energy ICRF driven minority ions and D-D fusion protons in JET using neutral particle analysis, in Proc. IAEA Technical Committee Meeting on Alpha Particles in Fusion Reseanch, Trieste 1993; Ya.I. Kolesnichenko and G.J. Sadler, Summary of IAEA technical committee meeting on alpha particles in fusion research, Trieste, Nucl. Fusion 33:1912 (1993). 